\title{
3D Garment Simulation and Visualization Based on Particle Spring Model
}

\author{
Mengjiao DONG ${ }^{1}, Y_{e}$ YUAN* \\ School of Information Engineering, Beijing Institute of Fashion Technology, Beijing, China \\ $1565315241 @ q q . c o m, g x y y y @ b i f t . e d u . c n$
}

\begin{abstract}
In view of the key technical problems that need to be solved in the digitization of 3D garment and garment tailoring, 3D garment simulation and visualization is mainly studied in this paper. Therefore, 3D clothing simulation and visualization based on particle spring model is proposed in this paper. Firstly, the fabric properties of the garment are briefly introduced, then the particle spring model and the collision detection algorithm are described. Finally, the algorithm is validated by selecting the human body model of each age group. The simulation results show that the proposed algorithm has certain effectiveness.
\end{abstract}

Keywords-fabric, particle spring, collision detection, 3D garment visualization

\section{INTRODUCTION}

The physical models commonly used in garment visual effects simulation are the mass grid model ${ }^{[1]}$, the particle system model ${ }^{[2-3]}$, and the particle spring model ${ }^{[4-5]}$. In order to make the cloth simulation results more realistic, the particle spring model is introduced in this paper. This model has been widely used in computer-aided 3D garment design, film and television special effects production, medical and games. Compared to the first two models, the particle spring model has the advantages of simple structure, easy to use algorithm and high efficiency. When simulating the real effect of $3 \mathrm{D}$ garment on the human body, a large number of collision phenomena are encountered: an interpenetration and traversal between the garment and the human body model and between the pieces themselves. Only when the problem of penetration and crossing is well solved can the simulation process of virtual dressing be realistically completed. Therefore, collision detection is the key to the entire simulation process.

\section{GARMENT FABRIC PERFORMANCE}

Fabric is a kind of flexible object. It is a very complicated physical system in both physical structure and mechanical behavior. The dynamic behavior of its physical model requires many parameters to be described from different angles. Because different fabrics are woven from a variety of fibers or yarns using different textile processes, and new textile products emerge in an endless stream, it is difficult to comprehensively collect and analyze the physical engineering, density and thickness properties of all fabrics. In the simulation of graphics, only a relatively simple model is used to simulate the representation rather than a comprehensive simulation of the physical properties of different texture fabrics. Therefore, this paper briefly introduces the complexity of the physical properties of fabrics, and extracts the common features of fabrics to prepare for the simulation experiments. In order to make the problem as simple as possible, it is assumed that the piece of the study is an ideal fabric having a certain quality and easy deformation, and the density and thickness of the fabric are assumed to be uniform. The current method for obtaining physical and mechanical properties of fabrics in the field of textiles is the Kawabata Evaluation System $^{[6]}$ for fabrics method. The method performs different types of tensile tests on the same piece of fabric, and the interactions within the fabric are uniformly classified into three types of stretching, shearing, and bending.

\section{MASS POINT SPRING MODEL}

The particle spring model is built according to the internal structure of the fabric and the external force. It can simulate 
the complex elastic deformation process and has a good effect on the static and dynamic performance of the flexible fabric.

In the modeling of $3 \mathrm{D}$ virtual garment technology, the triangular mesh is usually used to represent the topography of the fabric. The fabric is separated into a collection of particles and springs. The mass is evenly distributed on the particles, and the internal force is simplified to the elastic force between the particles. The particles are connected by springs according to certain rules. Generally speaking, there are three kinds of interactions inside the fabric: tension and compression, shearing and bending. The corresponding three kinds of springs are used to simulate these three modes of action: structural springs, twisted springs, stretchable spring as shown in Fig.1. The first is a structural spring that is used to connect the lateral and longitudinal particles to function as a fixed model structure. The second type is a twisted spring, also called a shear spring, that connects adjacent mass points on the diagonal to prevent distortion of the model. The third type is a tension spring, also called a bending spring, which connects two particle points separated by a mass point in the lateral and longitudinal directions to ensure that the edge of the model is rounded when deformed.

The algorithm for constructing a fabric model topology is as follows:

Step1: Establish the connection relationship between the spring and the particle.

Step2: Define the initial position and length of the spring.

Step3: Number each particle and spring according to a certain rule and establish a data structure.

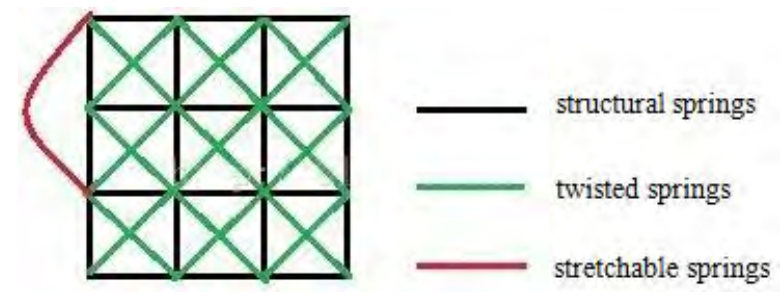

Fig.1. Particle Spring Model

After the particle spring model is established on the piece, the data points on the piece are no longer independent points that are unconnected, but interact by geometric and physical constraints of the spring.
During the simulation of the piece, all the particles on the piece produce a certain displacement due to the force. Calculate the resultant force of the mass by Newton's second law, as in formula (1).

$$
F_{\text {sum }}(i, j)=F_{\text {out }}(i, j)+F_{\text {in }}(i, j)=\operatorname{ma}(i, j)
$$

Among them, $F_{\text {sum }}(i, j)$ is the resultant force of the particle that is $\mathrm{P}\left(i_{s} j\right), F_{\text {out }}(i, j)$ is the external force received by the particle, $F_{\text {in }}(i, j)$ is the internal force received by the point, $\mathrm{m}$ is the quality of the point, and $a(i, j)$ is the acceleration of the point.

The force acting on the fabric can be divided into external force and internal force. The internal force is also called the spring force. The external force is the interaction between the fabric and the environment, including gravity, air resistance, and collision force, and the internal force includes the spring force and the damping force. In order to reduce the amount of calculation, this paper only considers the two external forces of gravity is $\mathrm{G}$ and air resistance is $F_{\mathrm{a}}(\mathrm{i}, j)$ as well as the spring force, as shown in formula (2).

$$
F_{\text {out }}(\mathrm{i}, j)=F_{\mathrm{a}}(\mathrm{i}, j)+G
$$

Among them, the gravity of the particle is as shown in the formula (3), and the air resistance of the particle is as shown in the formula (4).

$$
\mathrm{G}=\mathrm{m}(\mathrm{i}, \mathrm{j}) * \mathrm{~g}
$$

$$
\mathrm{F}_{\mathrm{a}}=-\mathrm{m}\left(\mathrm{i}_{\mathrm{s}} \mathrm{j}\right) * \mathrm{k}_{\mathrm{a}} * \mathrm{v}\left(\mathrm{i}_{\mathrm{j}} \mathrm{j}\right)
$$

Among them, $m\left(i_{3} j\right)$ is the quality of the particle that is $P\left(i_{x} j\right), g$ is the acceleration of gravity, is a constant value, which is set to 10 in the experiment. Since the quality distribution of the cloth is assumed to be uniform, the gravity 
of the particles is actually the same. $k_{a}$ is the normal number of defined air resistance, and $v(\mathrm{i}, j)$ is the velocity vector of the particle.

In the particle-spring model, the only elastic internal force considered is the elastic deformation force of the spring. Due to the ideal mass spring system, Hooke's law can be used to calculate the elastic deformation force of the spring, as in formula (5).

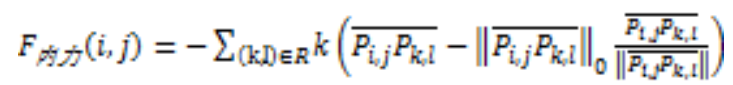

Among them, $\mathbf{k}$ is the elastic deformation coefficient of the spring, $\mathbb{R}$ is the set of adjacent points that is $\mathbb{P}_{i, j}$, $\left\|\overline{\mathrm{P}_{\mathrm{i}, j} \mathrm{P}_{\mathrm{k}, \mathrm{l}}}\right\|_{0}$ is the original distance between $\mathrm{P}_{\mathrm{i}, \overline{\mathrm{j}}}$ and $\mathrm{P}_{\left.\mathrm{k}_{\mathrm{j}}\right]}$, and $\mathrm{k}$ can be determined according to the material property parameter curve of the selected fabric.

In this paper, the Euler method is used to solve the fabric deformation model as in formula (6).

$$
\left\{\begin{array}{c}
a_{\mathrm{i}_{j} j}(t+\nabla t)=\frac{1}{m_{\mathrm{i}_{j} j}} F_{\mathrm{i}_{j} j}(t) \\
V_{\mathrm{i}_{j} j}(t+\nabla t)=V_{\mathrm{i}_{j} j}(t)+\nabla t a_{\mathrm{i}_{j} j}(t+\nabla t) \\
P_{\mathrm{i}_{i j} j}(t+\nabla t)=P_{\mathrm{i}_{j} j}(t)+\nabla t V_{\mathrm{i}_{j} j}(t+\nabla t)
\end{array}\right.
$$

Among them, $F_{i j j}$ is the resultant force of the mass point that is $P_{i, j}, m_{i j j}$ is the quality of the particle, $a_{i, j}(t), V_{i j}(t)$ and $\mathrm{P}_{\mathrm{i}, \mathrm{j}}(\mathrm{t})$ are the acceleration, velocity and displacement of the time of $t, \quad$ respectively, and $\nabla t$ is the time step.

\section{COLLISION DETECTION}

The core of the AABB based tree collision detection algorithm is to effectively traverse the two trees to determine whether some parts of the two collision bodies collide at the current position. This is a double recursive traversal process. The algorithm is described as follows:

Step1: Construct AABB trees for mannequins and pieces, respectively.

Step2: The root node of the AABB tree of the human body model traverses the AABB tree of the piece. If it is found that the bounding box of the root node of the manikin AABB tree does not intersect the bounding box of the inner node of the piece $\mathrm{AABB}$ tree, the downward traversal is stopped; if the traversal can reach the leaf node of the piece AABB tree, the leaf node is used again. Traverse the mannequin AABB tree. If the leaf nodes of the manikin AABB tree can be reached, the intersection test between the basic geometric elements is further performed.

Step3: Detects whether the basic geometric elements intersect.

The AABB model is traversed until the leaf nodes are traversed. Once the two bounding boxes are found to intersect, the geometric collision detection method is applied to test the collision between the triangles, and the self-collision of the pieces is detected by the same method. The collision detection between the two polygons is done by the intersection and proximity of the triangles of the checkpoints, similar to the collision detection between the piece and the mannequin. In order to avoid edge collision detection, the bounding box of the garment piece model is enlarged.

\section{SIMULATION RESULTS AND ANALYSIS}

Before performing the visual simulation of the garment, it is necessary to set the parameters of the particle-spring model for the force analysis, including the particle mass, gravity coefficient, spring coefficient and other parameters. The quality of fabrics with different materials is also different. For the convenience of calculation, the quality of the particles is set to 1 , and the degree of elastic deformation of the fabric is not more than 5\%. Only the elastic coefficient of the coefficient of the spring is considered. Refer to Kawabata. 
Experimental data, the elastic coefficient is between 0-20. In this paper, the spring modulus of the spring is set to a fixed value of 10 . The visual simulation algorithm for $3 \mathrm{D}$ clothing is as follows:

Step1: Discard the two-dimensional pieces and form the initial particle-spring system, and add various elastic forces according to the corresponding relationship between the particles. The pieces are scattered into a regular four-sided domain mesh, and the diagonal lines of the four-sided domain mesh are connected to form a spring particle system of a regular triangular mesh. The vertices of the triangle form a mass point, and the sides of the triangle form a corresponding spring. The triangulation of the pieces makes it easy to create AABB trees for the pieces.

Step2: Determine the combined force of the particles through mechanical analysis, and derive the acceleration of the particles from Newton's second law.

Step3: Calculate the velocity and displacement of the particle by Step2.

Step4: Repeat Step 2 to Step 3 to update the particle displacement until the displacement of each particle tends to a certain value, stop the operation, otherwise continue execution.

Step5: Establish AABB trees for human body models and pieces respectively. The two collision bodies involved in this paper are human body models and garment pieces, in which the human body model is static during the whole dynamic simulation process. Therefore, you only need to construct the $\mathrm{AABB}$ tree once at initialization.

Step6: Under the joint action of gravity and the internal elastic force between the mass points on the piece, the two-dimensional pieces will be gradually deformed and gradually spliced together. The whole splicing process is a dynamic iterative process. In the dynamic iterative process, a large number of collision detection processing between the human body model and the garment piece is carried out at the same time, and the corresponding collision response processing is given. After the splicing process is finished, the effect of the spliced three-dimensional garment on the static mannequin can be obtained.
In order to verify the validity and extensiveness of the algorithm, on the basis of this, three representative female human body models were selected in the simulation experiment, which were divided into three groups of young, middle and old, and the simulation was carried out to prove the practicality of the algorithm. Sex and effectiveness. The experimental human body model information is: a. The height of the young mannequin is $1.660 \mathrm{~m}$, the body weight is $52.8 \mathrm{~kg}$, which is thin; $b$. The height of the middle-aged human body model is $1.600 \mathrm{~m}$, the body weight is $54.4 \mathrm{~kg}$, which is normal type; c. The height of the elderly body model is $1.610 \mathrm{~m}$, the body weight $\mathrm{I}$ is $63.9 \mathrm{~kg}$, which is fatter. Simulation results are shown in Fig 2.

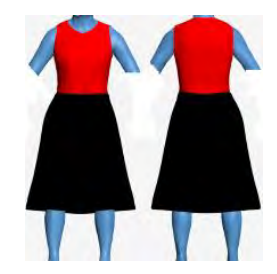

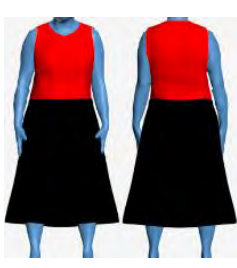

b

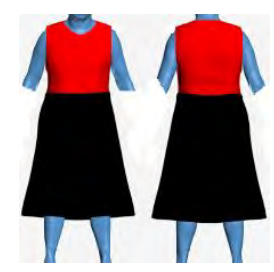

c
Fig.2. dressing effect

The simulation results show that the algorithm is applied to the 3D garment human body model, which is not affected by the factors such as high and low fat and thinness of the model. For different types of 3D garment human body models, the algorithm can achieve a good visual dressing effect.

\section{CONCLUSION}

The technologies of textile and garment, computer graphics and so on are used in this paper. Based on the research of garment prototype modeling, the particle spring model is used to simulate the virtual digital dressing of the 3D scanning human body model, and the clothing visualization is realized. The simulation results have achieved the expected results, indicating that the proposed algorithm has certain effectiveness.

\section{ACKNOWLEDGEMENT}

This work is supported by the science and technology project of Beijing Municipal Education Commission of 2017 with the granted No. SQKM201710012007 and also by the "Textile light" China Textile Industry Federation education 
and teaching reform project of 2017 with the granted No.

2017BKJGLX219.

\section{REFERENCES}

[1] $\mathrm{HN} \mathrm{Ng}, \mathrm{R}$ L Grimsdale, W G Allen. A system for modeling and visualization of cloth materials. Computer \& Graphics,1995, 19(3): 423-430

[2] Campo A D. Exact Quantum Decay of an Interacting Many-Particle System: the Calogero-Sutherland model[J]. Physics, 2015, 18(1).

[3] Plessas W. A model three-particle system for studying limitations of the shell-model approach to nuclear reactions[J]. Lecture Notes in Physics, 2006, 82:336-337.

[4] Zhang D, Whiten W J. The calculation of contact forces between particles using spring and damping models[J]. Powder Technology, 1996, 88(1):59-64.

[5] Raos G, Allegra G. Mesoscopic bead-and-spring model of hard spherical particles in a rubber matrix. I. Hydrodynamic reinforcement[J]. Journal of Chemical Physics, 2000, 113(17):7554-7563.

[6] TJ. Kang, WR. Yu. Drape simulation of woven fabric by using the finite element method[J]. Text.Inst,1995,86(4),635-647. 